\title{
HODGE THEORY WITH LOCAL COEFFICIENTS AND FUNDAMENTAL GROUPS OF VARIETIES
}

\author{
DONU ARAPURA
}

A pure R-Hodge structure of weight $n$ consists of a finite-dimensional real vector space $H$ together with a decomposition

$$
H \otimes \mathbf{C}=\bigoplus_{p+q=n} H^{p q}
$$

such that $\overline{H^{p q}}=H^{q p}$ (complex conjugation in $H \otimes \mathbf{C}$ is given by $\overline{v \otimes \alpha}=$ $v \otimes \bar{\alpha})$. We shall usually omit the prefix $\mathbf{R}$ in the sequel. The class of pure Hodge structures becomes a category, by declaring the morphisms to be $\mathbf{R}$-linear maps whose extensions to $\mathbf{C}$ preserve the decompositions. The fundamental example of pure Hodge structure of weight $n$ is provided by the $n$th real valued cohomology group of a complex smooth projective variety. This Hodge structure is constructed by representing cohomology classes by harmonic forms, with respect to a Kähler metric, and decomposing these forms into $(p, q)$ type.

For arbitrary complex varieties, Deligne has shown that the cohomology groups $H^{i}(X, \mathbf{R})$ carry a somewhat subtler decomposition called a mixed Hodge structure. Roughly speaking, a mixed Hodge structure is obtained by gluing together pure Hodge structures of different weights. The precise definition will not be given here (see [D1, 2.3]), instead we shall list a few basic properties. The collection of mixed Hodge structures constitute an abelian category which contains the category of pure Hodge structures as a full subcategory. Any mixed Hodge structure $H$ has a finite filtration (the weight filtration) $\cdots W_{i-1} \subset W_{i} \cdots$ by subobjects, such that each graded piece $\mathrm{Gr}_{i}^{W} H=W_{i} / W_{i-1}$ is pure of weight $i$. Weight filtrations are preserved by morphisms. In particular, subobjects of pure Hodge structures are also pure.

We have been able to generalize the above results, by constructing mixed Hodge structures on the cohomology of algebraic varieties with coefficients: locally constant sheaves arising from orthogonal representations of the fundamental group. Previously, Hodge theory with local coefficients was worked out by Deligne [Z, §2] for smooth projective varieties, and by Timmerscheidt $[\mathbf{T}]$ for arbitrary smooth varieties.

These results will be used to obtain information about fundamental groups of varieties. It is well known that the abelianization of the fundamental group of a smooth projective variety has even rank (this follows

Received by the editors December 21, 1988.

1980 Mathematics Subject Classification (1985 Revision). Primary 14F25, 14F45.

Partially supported by the National Science Foundation. 
from the Hodge decomposition $\left.H^{1}(X, \mathbf{R}) \otimes \mathbf{C}=H^{10} \oplus H^{01}\right)$. This simple observation admits the following generalization. Let $\mathbf{E}$ be the class of finitely presented groups $G$, such that given any real orthogonal representation $M$ (viewed as an $\mathbf{R}[G]$-module), $\operatorname{dim}_{\mathrm{R}} H^{1}(G, M)$ is even. Let $\mathbf{E}^{+}$be the class of groups $G$, all of whose subgroups of finite index lie in $\mathbf{E}$. We will show that the fundamental group of a compact normal variety lies in $\mathbf{E}^{+}$. As a consequence, we will show that the fundamental group of a compact normal variety cannot admit a decomposition $G * H$ with $G$ and $H$ finitely generated groups with nontrivial finite quotients. This was proved by Johnson and Rees [JR] for smooth projective varieties, by a different method.

THEOREM. Let $X$ be an algebraic variety over $\mathrm{C}$ and let $L$ be a locally constant sheaf of $\mathbf{R}$-vector spaces, with finite-dimensional stalks, equipped with a symmetric positive definite pairing $L \otimes L \rightarrow \mathbf{R}_{X}$. Then for all $i$, the cohomology groups $H^{i}(X, L)$ carry mixed Hodge structures, such that:

(a) The set $S=\left\{j \mid \mathrm{Gr}_{j}^{W} H^{i}(X, L) \neq 0\right\} \subseteq[0,2 i]$, furthermore $S \subseteq[0, i]$ if $X$ is compact and $S \subseteq[i, 2 i]$ if $X$ is smooth.

(b) If $f: Y \rightarrow X$ is a morphism of varieties then the maps

$$
H^{i}(X, L) \rightarrow H^{i}\left(Y, f^{*} L\right)
$$

are compatible with the mixed Hodge structures.

(c) If $L$ and $L^{\prime}$ are locally constant sheaves equipped with pairings as above, and if $g: L \rightarrow L^{\prime}$ is a map of sheaves (which need not preserve the pairings) then the maps

$$
H^{i}(X, L) \rightarrow H^{i}\left(X, L^{\prime}\right)
$$

are compatible with the mixed Hodge structures.

IDEA OF PROOF FOR COMPACT $X$. When $X$ is smooth and projective, the Hodge structure on $H^{i}(X, L)$ is constructed, as in the classical case, by harmonic forms. Let $V=L \otimes O_{X}$, this is a holomorphic vector bundle with an integrable connection $\nabla: A^{0}(V) \rightarrow A^{1}(V)$, where $A^{i}(V)$ (respectively $A^{p q}(V)$ ) denotes the space of $C^{\infty} V$-valued $i$-forms (respectively $(p, q)$ forms). Choose a Kähler metric on $X$. Using this metric and the pairing on $L$, an inner product can be placed on $A^{i}(V)$. An elliptic operator $\Delta$ on $A^{i}(V)$ can be constructed from $\nabla$ and its adjoint, such that $H^{i}(X, L) \cong$ ker $\Delta$. By the Kähler identities (in generalized form), the latter space carries a pure Hodge structure of weight $i$, with $H^{p q}=\operatorname{ker} \Delta \cap A^{p q}(V)$.

The general case is handled by the techniques of cohomological descent $[\mathrm{D2}, \S 5]$. A simplicial variety $X$. consists of a collection of varieties $\left\{X_{n}\right\}_{n \in \mathbf{N}}$ and maps $\partial_{i}: X_{n} \rightarrow X_{n-1}, s_{j}: X_{n-1} \rightarrow X_{n}(i=0, \ldots, n, j=$ $0, \ldots, n-1)$ satisfying certain identities [M, p. 1]. An augmentation of $X$. over $X$ is a collection of maps from $X_{n}$ to $X$ which commute with $\partial_{i}$ and $s_{j}$. Let $\Delta^{n}$ be the standard $n$-simplex in $\mathbf{R}^{n+1}, \delta_{i}: \Delta^{n-1} \rightarrow \Delta^{n}$ the inclusion of the $i$ th face, and $\sigma_{j}: \Delta^{n} \rightarrow \Delta^{n-1}$ the affine map which squashes the $j$ th and $(j+1)$ st vertices together. The geometric realization $|X$.$| , of a simplicial$ variety $X$., is the topological space obtained as a quotient of $\amalg X_{n} \times \Delta^{n}$ by the coarsest equivalence relation $\approx$ for which $\left(\partial_{i} x, t\right) \approx\left(x, \delta_{i} t\right)$ and 
$\left(s_{j} x, t\right) \approx\left(x, \sigma_{j} t\right)$. If $X$. is augmented over $X$ then there exists an induced continuous map $\pi:|X.| \rightarrow X ; X$. is said to be a hypercovering of $X$ if $\pi$ is a homotopy equivalence. (This is a little weaker than the usual definition.) Using resolution of singularities, one (i.e. Deligne) can construct a hypercovering of $X$, such that all the components $X_{n}$ are smooth and projective. Let $L_{n}$ denote the pullback of $L$ to $X_{n}$, then there is a spectral sequence

$$
E_{1}^{p q}=H^{q}\left(X_{p}, L_{p}\right) \Rightarrow H^{p+q}(X, L) .
$$

This will degenerate at $E_{2}$ (for rather subtle reasons). The spaces $E_{2}^{p q}$ carry pure Hodge structures of weight $q$, which are constructed as subquotients of the Hodge structures on $H^{q}\left(X_{p}, L_{p}\right)$. Then, crudely speaking, the mixed Hodge structure on $H^{i}(X, L)$ is obtained by gluing the pure Hodge structures on $E_{2}^{i 0}, E_{2}^{i-1,1} \ldots$. The actual construction proceeds on the level of complexes, it involves manufacturing a cohomological mixed Hodge complex on $X$. The details will be given elsewhere.

Corollary. Let $X$ be a compact normal variety then $\pi_{1}(X) \in \mathbf{E}^{+}$.

Proof. Since any finite unramified covering of $X$ is again a compact normal variety, it suffices to prove that $\pi_{1}(X) \in \mathbf{E}$. Let $M$ be an orthogonal real representation of $\pi_{1}(X)$ and let $L$ be the associated locally constant sheaf, then $L$ carries a symmetric positive definite pairing. We will show that $H^{1}(G, M)$ is even dimensional, by invoking the isomorphism $H^{1}(G, M) \cong H^{1}(X, L)$ [CE, p. 356 and $\mathrm{S}$, p. 360], and showing that the mixed Hodge structure on the latter space is of weight one. To do this, choose a desingularization $\pi: Y \rightarrow X$. The fibers of $\pi$ are connected [H, III 11.4], thus $\pi_{*} \pi^{*} L=L$. Consequently the map $H^{1}(X, L) \rightarrow H^{1}\left(Y, \pi^{*} L\right)$ is injective, since it can be identified with an edge homomorphism of the Leray spectral sequence

$$
E_{2}^{p q}=H^{p}\left(X, R^{q} \pi_{*} \pi^{*} L\right) \Rightarrow H^{p}\left(Y, \pi^{*} L\right) .
$$

By part (a), the mixed Hodge structure on $H^{1}\left(Y, \pi^{*} L\right)$ is pure of weight one, therefore the same is also true of $H^{1}(X, L)$.

We give some examples of groups which do not lie in $\mathbf{E}^{+}$and which therefore cannot be realized as fundamental groups of compact normal varieties.

LEMMA. (a) For any finitely generated group, the free product $\mathbf{Z} * G \notin \mathbf{E}^{+}$.

(b) If $G$ and $H$ are two finitely generated groups with nontrivial finite quotients then $G * H \notin \mathbf{E}^{+}$.

SKETCH OF PROof. (a) If rk $G /[G, G]$ is even then let $M$ be the trivial one-dimensional representation. Otherwise, let $M=\mathbf{R}$ with $G$ acting trivially and $\mathbf{Z}$ acting via $n \cdot x=(-1)^{n} x(n \in \mathbf{Z}, x \in \mathbf{R})$. In either case, one finds that $H^{1}(\mathrm{Z} * G, M)$ is odd dimensional.

(b) Using the Kurosh subgroup theorem, as in [JR, p. 465], one can find a subgroup, of finite index in $G * H$, of the form $F * K$, where $F$ is a nontrivial free group. Therefore by part (a), $G * H$ cannot lie in $\mathbf{E}^{+}$. 


\section{REFERENCES}

[CE] H. Cartan and S. Eilenberg, Homological algebra, Princeton Univ. Press, Princeton, N.J., 1956.

[D1] P. Deligne, Théorie de Hodge. II, Publ. Math. Inst. Hautes Études Sci. 40 (1971), $5-55$.

[D2] __, Théorie de Hodge. III, Publ. Math. Inst. Hautes Études Sci. 44 (1974), 5-77.

[H] R. Hartshorne, Algebraic geometry, Springer-Verlag, Berlin and New York, 1977.

[JR] F. E. A. Johnson and E. Rees, On the fundamental group of complex algebraic manifold, Bull. London Math. Soc. 19 (1987), 463-466.

[M] P. May, Simplicial objects in algebraic topology, Univ. of Chicago Press, Chicago, Ill., 1967.

[S] E. Spanier, Algebraic topology, McGraw-Hill, 1966.

[T] K. Timmerscheidt, Mixed Hodge theory for unitary local systems, J. Reine Angew. Math. 379 (1987), 152-171.

[Z.] S. Zucker, Hodge theory with degenerating coefficients, Ann. of Math. (2) 109 (1979), 415-476.

Department of Mathematics, Purdue University, West Lafayette, Indiana 47907

Mathematical Sciences Research Institute, 1000 Centennial Drive, Berkeley, CALIFORNIA 94720 